\title{
In Situ TEM Studies on the Nucleation and Growth of Multicomponent Alloy Nanoparticles on 2D Materials
}

\author{
Mahmoud Tamadoni Saray ${ }^{1}$ and Reza Shahbazian-Yassar ${ }^{2}$ \\ ${ }^{1}$ University of Illinois at Chicago (UIC), Chicago, Illinois, United States, ${ }^{2}$ University of Illinois at Chicago, \\ Chicago, Illinois, United States
}

How could we overcome catalytic and electrochemical performance decay dilemma in conductive host supported nanoparticles systems? To answer this question, we come up with concept of implementing emerging high entropy alloy (HEA) merits to synthesize high quality and well dispersed multi-element mixed NPs on reduced graphene oxide nanosheets with dry green chemistry high temperature shock (HTS) approach and in situ monitoring the dynamic formation process with atomic level characterization using transition electron microscopy (TEM). The earlier provides the synergistic effects and solid solution advantages of atomically mixed multi principal elements of HEA NPs on conductive carbon substrate to overcome conventional intrinsic complexity of multi-element mixing at nanoscale while the later delivers direct precise high spatial resolution information regarding the formation kinetics/mechanism and stabilization of HEA NPs [1-3]. We simulated HTS with an electrical biasing in situ TEM set up to observe IrPtRuFeNi HEA NPs from mixed metal precursor salts on graphene oxide. We assume that graphene oxide functional groups/active sites might act as nucleation sites for NPs formation during shocking metal salt covered graphene oxide nanosheets. It is worth mentioning that while high temperature $(1200 \mathrm{C}, 2 \mathrm{sec})$ not only decomposes metal salts but also contributes to fast atomic interdiffusion and uniform dispersion of metal atoms within NPs, fast quenching step to room temperature secures homogenous composition distribution of metal atoms within the HEA NPs.

In this study, structural evolution monitored via in situ TEM, chemical characterization extracted via aberration corrected STEM/HRTEM, selected area electron diffraction (SAED), energy-dispersive X-ray spectroscopy (EDS), and Electron Energy Loss Spectroscopy (EELS) confirmed solid solution IrPtRuFeNi HEA NPs with single cubic phase crystal structure. Very short heating time (i.e., few sec) and the strong bonding between the formed HEA NPs and reduced graphene nanosheets suppress the migration and subsequent agglomeration phenomena which is very critical especially under high temperature condition applications. Uniformly metal salts covered graphene oxide nanosheets (Figure 1a) transforms to anchored nanosized HEA NPs with narrow size distribution after quick HTS (Figure 1b). Well resolved lattice fringes via HRTEM and Fourier transfer (FFT) confirmed structural uniformity of the synthesized faceted HEA NPs (Figure 1c, d) which will be promising for catalytic performance. Moreover, highly random dispersion of five metal signals in EDS elemental mappings verified chemical uniformity (Figure 2).

Therefore, our focus is to throwing light on the underlying formation/anchoring mechanisms, structural chemistry/stability, phase transformation, size, composition, morphology, interactions among multiple metal cations and carbonaceous substrate of this new entropy driven materials with fine features through different precursor salts ratio, heating conditions via powerful in situ TEM which will be beneficial for rapid, energy efficient clean bulk HEA NPs hybrid synthesis for advanced energy storage and catalytic applications. 

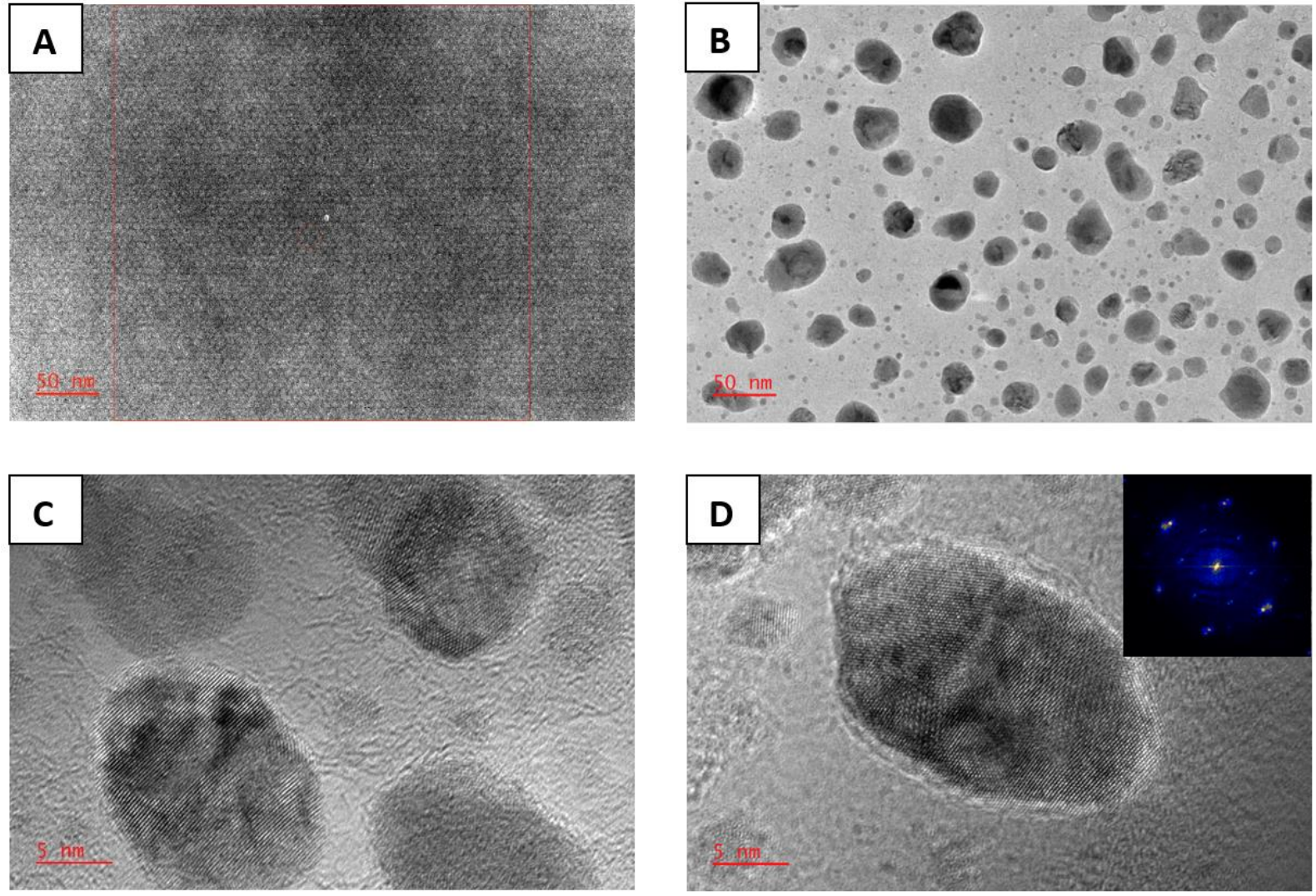

Figure 1. (A) Low magnification TEM image of metal salts covered graphene oxide nanosheets (B) Low magnification TEM image of HEA NPs formed after HTS. (C, D) HTEM images of HEA NPs on reduced graphene oxide nanosheets [inset in D is FFT of the image].
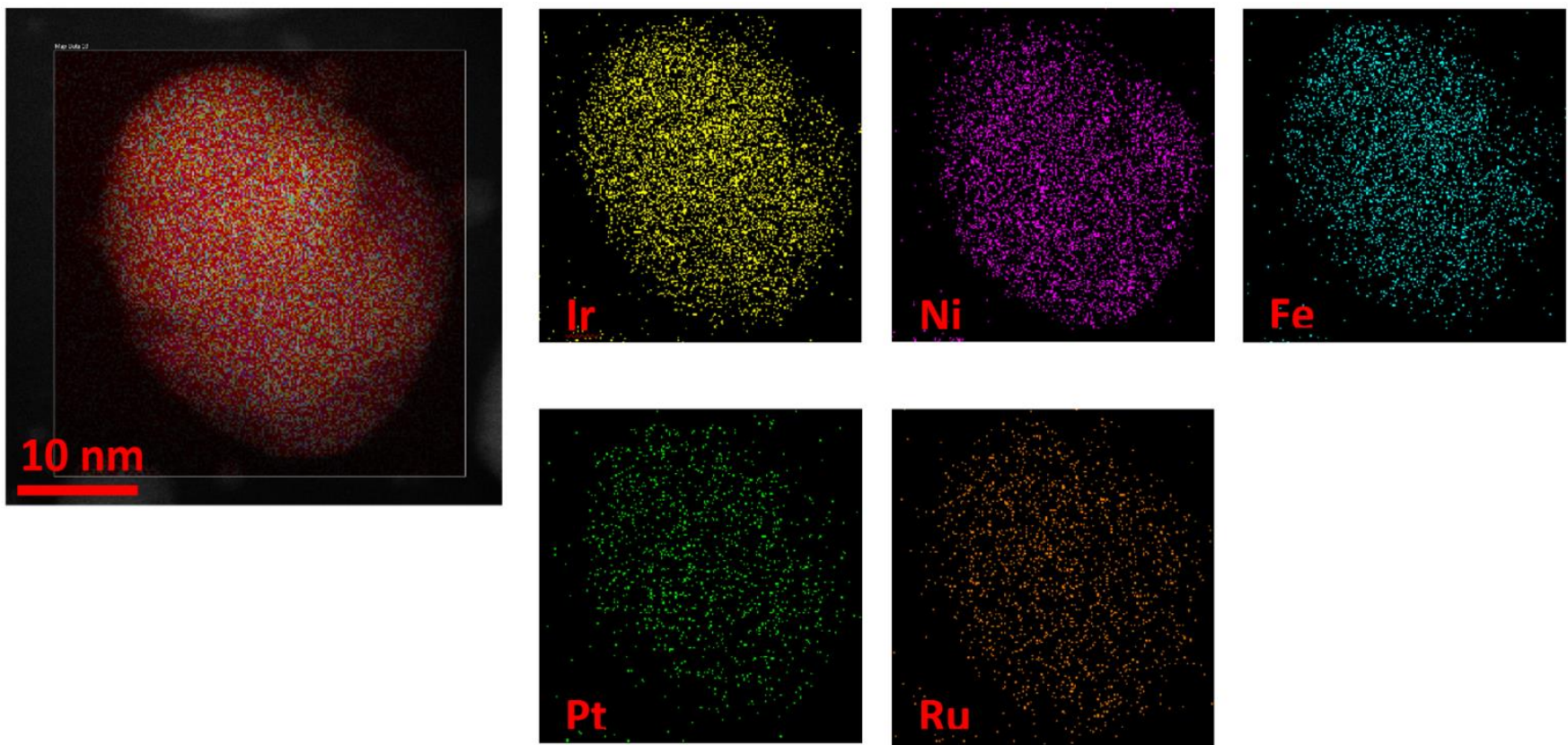

Figure 2. EDS elemental mapping images of Ir, Ni, Fe, Pt, Ru in HTS synthesized HEA NP. 


\section{References}

[1] E. P. George et al., High-entropy alloys. Nature Reviews Materials, 4 (2019), p. 515-534.

[2] Y, Yuan et al., Understanding materials challenges for rechargeable ion batteries with in situ transmission electron microscopy. Nature Communications. 8 (2017), p.15806.

[3] Z, Huang et al., Direct observation of the formation and stabilization of metallic nanoparticles on carbon supports. Nature communications 11 (2020), p. 1-9.

[4] This work made use of instruments in the Electron Microscopy Service (Research Resources Center, UIC). R.S.Y acknowledges financial support from the National Science Foundation (DMR-1809439). 